\title{
The temperature dependence of the inhibition of growth of a protozoan by antibiotics*
}

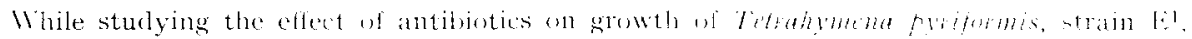
it was decided to extend kinetic experinents to detemine the response the vared indulation

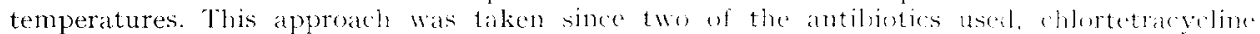

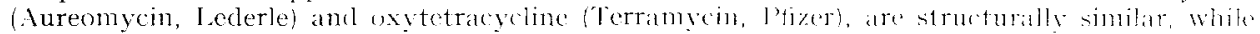
the third drug, chlomphenicol (Chormmcetin, Parke, Davis) is different. It was hepock. therefore, that this type of investigation might help to discriminate betwern the modes of action of these compounclit.

Culture technics, chemically detined and stock media, solution preparations and the turbiclimetric method of growth measurenent were the same as those previously reported", with two exceptions: (I) the defined medium was modified by the alse of the grow th factor, thioctic acid ${ }^{*}$, in place of protogen 1 ; and (2) experimental cultures were grown at the average temperatures of 28,25 (comtrol), If and of C, respectively, with a variation 1) +1.5 (c in each asse.

Final antibiotic concentrations of 3.1 .

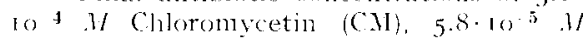
Sureomycin (AM) and 1.5. I0 t M Terramyein (ТМ) were employed, because these concentrations were fom to inhibit growth rate by $50^{\circ}$, at the control temperaturel, 2 .

The intuence of temperature on growth 1) Tetrahyena is illustrated in ligg. I. The slope, m, of the growth curve was calculated from the formula: $n$ I $\quad$ og $d_{1} \ldots . .$. Iog $d_{2}$. $t_{1}-t_{2}$

where $d$ - optical density, and $t-t^{2}$ time in lays. It is obvious from this plot, that as temperature decreases (I) growth rate (stope) does likewise, (2) peak growth increases and (3) logarithmic growth phase becomes prolonged. These are the expected effer ts in invertebrates in general . lowered temperatures for growth usually result in acceased metabolic rate, increased longevity and larger size or borly weight

The relative effect of the nomally $50^{\circ}$ suppressive levels tat 25 Cy of the antibiotics is shown in relation to temperature in Iig. 2. Results are plotted as the calculated slope, $m$, against the reciprocal of the alsolute temperature, T. Chief!y, two results are noteworthy. Firstly, as temperature is lowerel, the activity of each of the drugs is decreased until at $16 \mathrm{C}$, growth rates of control and antibiotic-containing cultures are essentially equal. In this respect, the same general
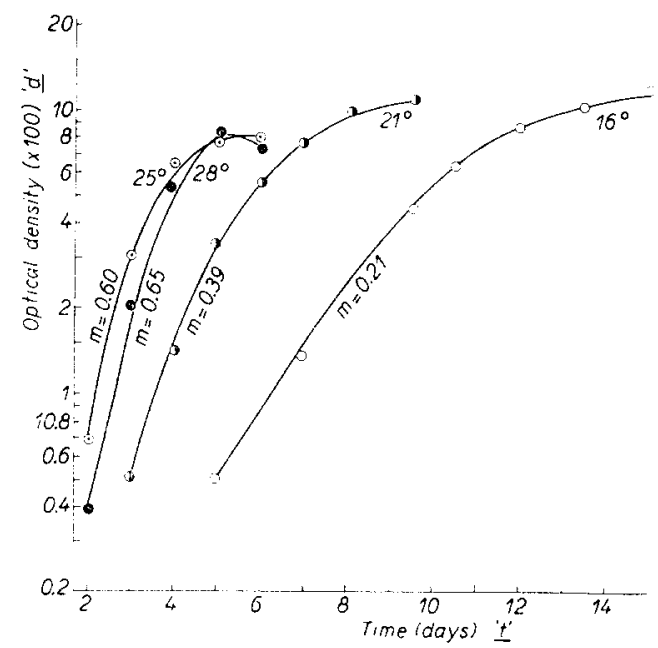

Fig. 1. Growth of Teballwmene in relation to temperature ( C). m - slope (rate of growth): $d$-. optical density $t \ldots$ days.

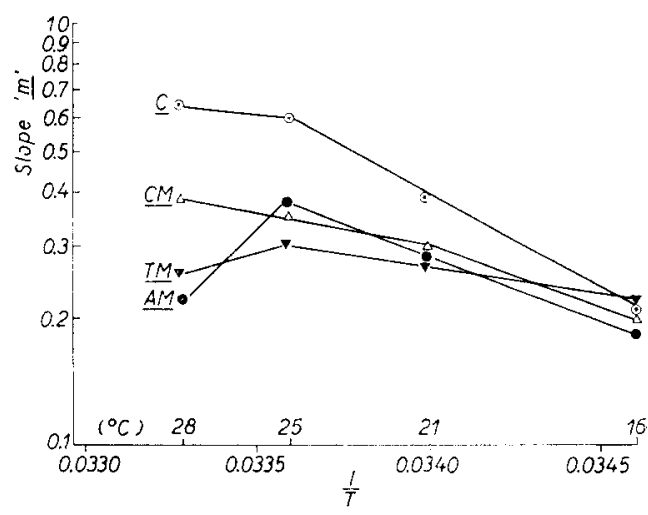

lig. 2. Interaction of antibiotics and temperature on growth rate of Tetrahymena. $C=$ control: $\mathrm{CM}=$ Chloromycetin: TM = Terramycin: $\mathrm{AM}=$

Aureomycin: $T=$ absolute temperature. picture is revealed for the action of all three compouncls, in. potency decrease with decrease in temperature, regardless of their respective chemica! structures. Secondly, thore is an indication fron the shapes of the plotted lines, that

* From a dissertation submitted in partial fulfilment of the requirements for the degree of Doctor of Philosophy in the Iniversity of Michigan, 1953.

* Thioctic acid, as Na-D,L-6-thioctate, was supplied by Dr. L. L. R. Srokstad w leederte? laboratories, Pearl River, X.Y. 
the two tetracyclines, $A M$ and $T M$, react similarly, while $C M$, which is structurally dissimilar, acts differently. It appears, therefore, that a study such as that described is able to distinguish between compounds with like and unlike modes of action.

Following the analytical precedent set by JoHnson et al.$^{3}$, these antibiotics appear qualitatively to fall into the Type II class of inhibitors ${ }^{4}, 5$, i.e. their activity declines with clecrease in temperature. All observed Type II inhibitors are also known to be biochemically "noncompetitive".

Furthermore, Woolley ${ }^{6}$ has demonstrated, that in Escherichia coli, CM acts as a noncompetitive inhibitor. The data shown in this report, then, uphold Wooldey's contention and in addition indicate that TM and AM function in the same manner. Therefore, it is tentatively concluded that the general mechanism of action of the three antibiotics is the same in that they are Type II inhibitors. However, their specific mechanisms or sites of action seem to be different. and appear to Jepend upon their chemical structure.

A more critical analysis of these results is untenable at this time. Such analysis must await further studies at intermediate temperatures and varied antibiotic concentrations, as well as growth carried out in constant temperature chambers or water baths of greater sensitivity than those utilized in this investigation.

My gratitude is expressed to Dr. D. E. S. Brown for suggesting this study, and to Dr. A, M. ELLJOTT under whose direction the investigation was performed.

Department of Zoology, University of Michigan, Ann Arbor, Mich. (U.S.A.)

J. A. Gross* ${ }^{* *}$

1 J. A. Gross, Ph. D. thesis (1953).

2 J. A. Gross, J. Protozool., 2 (1955) 42.

3 F. H. Johnson, H. Eyring and R. W. Williams, J. Cellular Comp. Physiol., 20 (1942) 247.

4 W. D. Mc Elroy, J. Cellular Comp. Physiol., 2 I (1943) 95.

5 W. D. McElroy, Quart. Rev. Biol., 22 (1947) 25.

6 D. W. Woolley, J. Biol. Chem., I85 (1950) 293.

Received August 23rd, I955

* Predoctoral Research Fellow of the United States Atomic Energy Commission, I95I-53.

** Present address: Zoology Department, I Iniversity of California, Los Angeles 24, California, IJ.S.A.

\section{A sensitive reaction on paper of ninhydrin with hydroxyproline and proline}

The sensitivity of the ninhydrin (triketo-hydrindene hydrate) reaction with amino acids separated on two dimensional paper chromatograms has been described by PRATT AND AUCLAIR ${ }^{1}$. The reagent has a low sensitivity for both hydroxy-proline and proline due to the formation of a yellow pigment which is difficult to differentiate from the white paper background.

A more satisfactory qualitative test was evolved using the following procedure. The filter paper chromatograms, after development with the solvent system of REDFIELD ${ }^{2}$ and steaming for ro minutes to remove adsorbed diethylamine, were dipped in a $0.1 \%(\mathrm{w} / \mathrm{v})$ solution of ninhydrin in benzene containing $0.1 \%(\mathrm{v} / \mathrm{v})$ collidine. The papers were then placed in dry steam for I minute to produce magenta spots from hydroxy-proline and proline, which exhibited an intense red fluorescence in $\mathrm{u} . \mathrm{v}$. light. The limit of detection by $\mathrm{u} . \mathrm{v}$. fluorescence was $0.008 \mu \mathrm{g}$ and $0.025 \mu \mathrm{g}$ amino-nitrogen for hydroxy-proline and proline respectively.

A pigment of similar colour is known to occur as an intermediate in the hydroxy-proline ninhydrin reaction ${ }^{3}$. This substance, subsequently referred to as pigment $A$, can be isolated from the aqueous reaction media by extraction with benzene ${ }^{4}$, and is non-fluorescent in $u$.v. light. It has an absorption spectrum in the $35^{\circ}$ to $75^{\circ} \mathrm{m} / \mathrm{range}$ identical with that of the fluorescent pigment (pigment B) as measured whilst adsorbed on the paper. This was achieved by placing. paper strips carrying the pigment in a glass cuvette containing benzene. Pigments $A$ and $B$ both exhibit an absorption maximum at $570 \mathrm{~m} \mu$ and similar strong absorption $260-280 \mathrm{~m} \mu$. Pigment $B$ was found to be inextractable from the paper by both polar and non-polar solvents except to a small extent by acetone. The latter gave a magenta-coloured solution, non-fluorescent in u.v. light with absorption spectrum identical with that of pigment $A$ in acetone-each maximal at $55^{\circ} \mathrm{m} \mu$. This suggests that $B$ is formed by adsorption of $A$ on the paper and this is supported by the observation that addition of $\mathrm{A}$ to the paper produced a magenta spot which became fluorescent on steaming for one minute. Moreover, both A and B on prolonged heating yielded a yellow pigment of identical absorption spectra with that published by MOORE AND STEIN ${ }^{5}$. 